Pawel Hanus and Mariusz Urbański, Department of Mathematics, University of North Texas, Denton, TX 76203-5118. e-mail: urbanski@unt.edu

\title{
RIGIDITY OF INFINITE ONE-DIMENSIONAL ITERATED FUNCTION SYSTEMS
}

\begin{abstract}
In $[\mathrm{MU}]$ the concept of infinite conformal iterated function systems was introduced and developed. In this paper we consider 1-dimensional systems. We provide necessary and sufficient conditions for such systems to be bi-Lipschitz equivalent. We extend to such systems the concept of scaling functions and we pay special attention to the real-analytic systems.
\end{abstract}

\section{Preliminaries.}

In $[\mathrm{MU}]$ we provided the framework to study infinite conformal iterated function systems. Let us recall this notion assuming that $X$ is a 1-dimensional interval. Let $I$ be a countable index set with at least two elements and let $S=\left\{\phi_{i}: X \rightarrow X: i \in I\right\}$ be a collection of injective contractions from X into $\mathrm{X}$ for which there exists $0<s<1$ such that $\rho\left(\phi_{i}(x), \phi_{i}(y)\right) \leq s \rho(x, y)$ for every $i \in I$ and for every pair of points $x, y \in X$. Thus, the system $S$ is uniformly contractive. Any such collection $S$ of contractions is called an iterated function system. We are particularly interested in the properties of the limit set defined by such a system. We can define this set as the image of the coding space under a coding map as follows. Let $I^{*}=\bigcup_{n>1} I^{n}$, the space of finite words, and for $\omega \in I^{n}, n \geq 1$, let $\phi_{\omega}=\phi_{\omega_{1}} \circ \phi_{\omega_{2}} \circ \cdots \circ \phi_{\omega_{n}}$. If $\omega \in I^{*} \cup I^{\infty}$ and $n \geq 1$ does not exceed the length of $\omega$, we denote by $\left.\omega\right|_{n}$ the word $\omega_{1} \omega_{2} \ldots \omega_{n}$. Since given $\omega \in I^{\infty}$, the diameters of the compact sets $\phi_{\omega \mid n}(X), n \geq 1$, converge to zero and since they form a descending family, the

Mathematical Reviews subject classification: 26A18, 28D05, 58F03, 58F11 and 58F12

Received by the editors September 9, 1997

*Partially supported by TARP Grant 003594-017 
set $\bigcap_{n=0}^{\infty} \phi_{\left.\omega\right|_{n}}(X)$ is a singleton and therefore, denoting its only element by $\pi(\omega)$, defines the coding map $\pi: I^{\infty} \rightarrow X$. The main object of our interest will be the limit set

$$
J=\pi\left(I^{\infty}\right)=\bigcup_{\omega \in I^{\infty}} \bigcap_{n=1}^{\infty} \phi_{\omega \mid n}(X) .
$$

Observe that $J$ satisfies the natural invariance equality, $J=\bigcup_{i \in I} \phi_{i}(J)$. Notice that if $I$ is finite, then $J$ is compact and this property fails for infinite systems.

Definition. An iterated function system $S=\left\{\phi_{i}: X \rightarrow X: i \in I\right\}$ is said to satisfy the Open Set Condition if there exists a nonempty open set $U \subset X$ (in the topology of X) such that $\phi_{i}(U) \subset U$ for every $i \in I$ and $\phi_{i}(U) \cap \phi_{j}(U)=\emptyset$ for every pair $i, j \in I, i \neq j$.

An iterated function system $S$ satisfying the Open Set Condition is said to be conformal (c.i.f.s.) if the following conditions are satisfied.

(a) $U=\operatorname{int}(X)$.

(b) There exists an open connected set $X \subset V \subset \mathbb{R}$ such that all maps $\phi_{i}$, $i \in I$, extend to $C^{1}$ diffeomorphisms of $V$ into $V$.

(c) Bounded Distortion Property(BDP). There exists $K \geq 1$ such that

$$
\left|\phi_{\omega}^{\prime}(y)\right| \leq K\left|\phi_{\omega}^{\prime}(x)\right|
$$

for every $\omega \in I^{*}$ and every pair of points $x, y \in V$, where $\left|\phi_{\omega}^{\prime}(x)\right|$ means the norm of the derivative.

For simplicity and clarity of our exposition we assumed the open set $U$ appearing in the open set condition is $\operatorname{int}(X)$. As was demonstrated in [MU], conformal iterated function systems naturally break into two main classes, irregular and regular. This dichotomy can be determined from either the existence of a zero of a natural pressure function or, equivalently, the existence of a conformal measure. The topological pressure function, $P$ is defined as follows. For every integer $n \geq 1$ let

$$
\psi_{n}(t)=\sum_{\omega \in I^{n}}\left\|\phi_{\omega}^{\prime}\right\|^{t} \text { and } P(t)=\lim _{n \rightarrow \infty} \frac{1}{n} \log \psi_{n}(t) .
$$

For a conformal system $S$, we sometimes set $\psi_{S}=\psi_{1}=\psi$. The finiteness parameter, $\theta_{S}$, of the system $S$ is defined by $\inf \{t: \psi(t)<\infty\}=\theta_{S}$. In [MU], 
it was shown that the topological pressure function $P(t)$ is non-increasing on $[0, \infty)$, strictly decreasing, continuous and convex on $[\theta, \infty)$ and $P(1) \leq 0$. Of course, $P(0)=\infty$ if and only if $I$ is infinite. In [MU] (see Theorem 3.15) we have proved the following characterization of the Hausdorff dimension of the limit set $J$, which will be denoted by $\operatorname{HD}(J)=h_{S}$.

Theorem 1.1. $\operatorname{HD}(J)=\sup \left\{\operatorname{HD}\left(J_{F}\right): F \subset I\right.$ is finite $\}=\inf \{t: P(t) \leq 0\}$. If $P(t)=0$, then $t=\mathrm{HD}(J)$.

We called the system $S$ regular provided that there is some $t$ such that $P(t)=0$. It follows from [MU] that $t$ is unique. Also, the system is regular if and only if there is a $t$-conformal measure. Recall that a Borel probability measure $m$ is said to be $t$-conformal provided $m(J)=1$ and for every Borel set $A \subset X$ and every $i \in I$

$$
m\left(\phi_{i}(A)\right)=\int_{A}\left|\phi_{i}^{\prime}\right|^{t} d m \text { and } m\left(\phi_{i}(X) \cap \phi_{j}(X)\right)=0,
$$

for every pair $i, j \in I, i \neq j$. We recall also (see [MU, Theorem 3.8]) that there exists an invariant measure $\mu$ (in the sense that for every measurable set $A, \mu\left(\bigcup_{i \in \mathbb{N}} \phi_{i}(A)\right)=\mu(A)$ equivalent with $m$ and $d \mu / d m$ is bounded away from zero and infinity.

Finally, we call two iterated function systems $\left\{f_{i}: X \rightarrow X, i \in \mathbb{N}\right\}$ and $\left\{g_{i}: Y \rightarrow Y, i \in \mathbb{N}\right\}$ topologically conjugate if and only if there exists a homeomorphism $h: J_{F} \rightarrow J_{G}$ such that $h \circ f_{i}=g_{i} \circ h$ for all $i \in \mathbb{N}$. Then by induction we easily get that $h \circ f_{\omega}=g_{\omega} \circ h$ for every finite word $\omega$.

\section{General Systems.}

The main result of this section is the following assertion.

Theorem 2.1. Suppose that $F=\left\{f_{i}: X \rightarrow X, i \in \mathbb{N}\right)$ and $G=\left\{g_{i}: Y \rightarrow\right.$ $Y, i \in \mathbb{N}\}$ are two topologically conjugate one-dimensional conformal iterated function systems. Then the following 4 conditions are equivalent.

(1) $\exists S \geq 1 \forall \omega \in \mathbb{N}^{*}$

$$
S^{-1} \leq \frac{\operatorname{diam}\left(g_{\omega}(Y)\right)}{\operatorname{diam}\left(f_{\omega}(X)\right)} \leq S .
$$

(2) $\left|g_{\omega}^{\prime}\left(y_{\omega}\right)\right|=\left|f_{\omega}^{\prime}\left(x_{\omega}\right)\right|$ for all $\omega \in \mathbb{N}^{*}$, where $x_{\omega}$ and $y_{\omega}$ are the only fixed points of $f_{\omega}: X \rightarrow X$ and $g_{\omega}: Y \rightarrow Y$ respectively.

(3) $\exists E \geq 1 \forall \omega \in \mathbb{N}^{*} E^{-1} \leq \frac{\left\|g_{\omega}^{\prime}\right\|}{\left\|f_{\omega}^{\prime}\right\|} \leq E$. 
(4) For every finite subset $T$ of $\mathbb{N}, \operatorname{HD}\left(J_{G, T}\right)=\operatorname{HD}\left(J_{F, T}\right)$ and the conformal measures $m_{G, T}$ and $m_{F, T} \circ h^{-1}$ are equivalent.

Suppose additionally that both systems $F$ and $G$ are regular. Then the following condition is also equivalent with the four conditions above.

(5) $\operatorname{HD}\left(J_{G}\right)=\operatorname{HD}\left(J_{F}\right)$ and the conformal measures $m_{G}$ and $m_{F} \circ h^{-1}$ are equivalent.

Proof. Let us first demonstrate that conditions (2) and (3) are equivalent. Indeed, suppose that (2) is satisfied and let $K_{F}$ and $K_{G}$ denote the distortion constants of the systems $F$ and $G$ respectively. Then for all $\omega \in \mathbb{N}^{*},\left\|g_{\omega}^{\prime}\right\| \leq$ $K_{G}\left|g_{\omega}^{\prime}\left(y_{\omega}\right)\right|=K_{G}\left|f_{\omega}^{\prime}\left(x_{\omega}\right)\right| \leq K_{G}\left\|f_{\omega}^{\prime}\right\|$ and similarly $\left\|f_{\omega}^{\prime}\right\| \leq K_{F}\left\|g_{\omega}^{\prime}\right\|$. So suppose that (3) holds and (2) fails, that is that there exists $\omega \in \mathbb{N}^{*}$ such that $\left|g_{\omega}^{\prime}\left(y_{\omega}\right)\right| \neq\left|f_{\omega}^{\prime}\left(x_{\omega}\right)\right|$. Without lose of generality we may assume that $\left|g_{\omega}^{\prime}\left(y_{\omega}\right)\right|<\left|f_{\omega}^{\prime}\left(x_{\omega}\right)\right|$. For every $n \geq 1$ let $\omega^{n}$ be the concatenation of $n$ words $\omega$. Then $g_{\omega^{n}}\left(y_{\omega}\right)=g_{\omega}^{n}\left(y_{\omega}\right)=y_{\omega}$ and similarly $f_{\omega^{n}}\left(x_{\omega}\right)=x_{\omega}$. So, $x_{\omega^{n}}=$ $x_{\omega}=\pi_{F}\left(\omega^{\infty}\right)$ and $y_{\omega^{n}}=y_{\omega}=\pi_{G}\left(\omega^{\infty}\right)$. Moreover $\left|g_{\omega^{n}}^{\prime}\left(y_{\omega}\right)\right|=\left|g_{\omega}^{\prime}\left(y_{\omega}\right)\right|^{n}$ and $\left|f_{\omega^{n}}^{\prime}\left(x_{\omega}\right)\right|=\left|f_{\omega}^{\prime}\left(x_{\omega}\right)\right|^{n}$. Hence

$$
\lim _{n \rightarrow \infty} \frac{\left|g_{\omega^{n}}^{\prime}\left(y_{\omega}\right)\right|}{\left|f_{\omega^{n}}^{\prime}\left(x_{\omega}\right)\right|}=0
$$

On the other hand, by (3) and the Bounded Distortion Property

$$
\frac{\left|g_{\omega^{n}}^{\prime}\left(y_{\omega}\right)\right|}{\left|f_{\omega^{n}}^{\prime}\left(x_{\omega}\right)\right|} \geq \frac{K_{G}^{-1}|| g_{\omega^{n}}^{\prime} \|}{\left\|f_{\omega^{n}}^{\prime}\right\|} \geq E^{-1} K_{G}^{-1}
$$

for all $n \geq 1$. This contradiction finishes the proof of equivalence of conditions (2) and (3). Since the equivalence of (1) and (3) is immediate, the proof of the equivalence of conditions (1)-(3) is finished.

We shall now prove that $(3) \Rightarrow(5)$. Indeed, it follows from (3) that $E^{-1} \psi_{G, n}(t) \leq \psi_{F, n}(t) \leq E \psi_{G, n}(t)$ for all $t \geq 0$ and all $n \geq 1$. Hence $P_{G}(t)=P_{F}(t)$ and therefore by Theorem 1.1, $\operatorname{HD}\left(J_{G}\right)=\operatorname{HD}\left(J_{F}\right)$. Denote this common value by $h$. Although we keep the same symbol for the homeomorphism establishing conjugacy between the systems $F$ and $G$, it will never cause misunderstandings. Suppose now that both systems are regular (in fact assuming (3) regularity of one of these systems implies regularity of the other). Then for every $\omega \in \mathbb{N}^{*}$

$$
\left(K_{F} E\right)^{-h} \leq \frac{K_{F}^{-h}\left\|f_{\omega}^{\prime}\right\|^{h}}{\left\|g_{\omega}^{\prime}\right\|^{h}} \leq \frac{m_{F}\left(f_{\omega}\left(J_{F}\right)\right)}{m_{G}\left(g_{\omega}\left(J_{G}\right)\right)} \leq \frac{\left\|f_{\omega}^{\prime}\right\|^{h}}{K_{G}^{-h}\left\|g_{\omega}^{\prime}\right\|^{h}} \leq\left(E K_{G}\right)^{h} .
$$


So, the measures $m_{G}$ and $m_{F} \circ h^{-1}$ are equivalent, and even more

$$
\left(K_{F} E\right)^{-h} \leq \frac{d m_{F} \circ h^{-1}}{d m_{G}} \leq\left(E K_{G}\right)^{h} .
$$

Let us show now that $(5) \Rightarrow(3)$. Indeed, if (5) is satisfied, then the measure $\mu_{F} \circ h^{-1}$ is equivalent with $\mu_{G}$. Since additionally $\mu_{F} \circ h^{-1}$ and $\mu_{G}$ are both ergodic (see Theorem 3.8 of [MU]), they are equal. Hence, using the equality $\operatorname{HD}\left(J_{F}\right)=\operatorname{HD}\left(J_{G}\right):=h$, we get

$$
\begin{aligned}
\left\|g_{\omega}\right\|^{h} & \asymp \int\left|g_{\omega}^{\prime}\right|^{h} d m_{G}=m_{G}\left(g_{\omega}\left(J_{G}\right)\right) \asymp \mu_{G}\left(g_{\omega}\left(J_{G}\right)\right) \\
& =\mu_{F} \circ h^{-1}\left(g_{\omega}\left(J_{G}\right)\right)=\mu_{F}\left(f_{\omega}\left(J_{F}\right)\right) \asymp m_{F}\left(f_{\omega}\left(J_{F}\right)\right) \\
& =\int\left|f_{\omega}^{\prime}\right|^{h} d m_{F} \asymp\left\|f_{\omega}^{\prime}\right\|^{h}
\end{aligned}
$$

and raising the first and the last terms of this sequence of comparabilities to the power $1 / h$, we finish the proof of the implication $(5) \Rightarrow(3)$.

The equivalence of (4) and conditions (1) - (3) is now a relatively simple corollary. Indeed, to prove that (3) implies (4) fix a finite subset $T$ of $\mathbb{N}$. By (3) $E^{-1} \leq\left\|f_{\omega}^{\prime}\right\| /\left\|g_{\omega}^{\prime}\right\| \leq E$ for all $\omega \in T^{*}$, and as every finite system is regular, the equivalence of measures $m_{G, T}$ and $m_{F, T} \circ h^{-1}$ follows from the equivalence of conditions (3) and (5) applied to the systems $\left\{f_{i}: i \in T\right\}$ and $\left\{g_{i}: i \in T\right\}$. If in turn (4) holds and $\omega \in \mathbb{N}^{*}$, then $\omega \in T^{*}$, where $T$ is the (finite) set of letters making up the word $\omega$ and the measures $m_{G, T}$ and $m_{F, T} \circ h^{-1}$ are equivalent. Hence, by the equivalence of (2) and (5) applied to the systems $\left\{f_{i}: i \in T\right\}$ and $\left\{g_{i}: i \in T\right\}$ we conclude that $\left|g_{\omega}^{\prime}\left(y_{\omega}\right)\right|=\left|f_{\omega}^{\prime}\left(x_{\omega}\right)\right|$. Thus (2) follows and the proof of Theorem 2.1 is finished.

We say that a conformal system $\left\{\phi_{i}: X \rightarrow X: i \in \mathbb{N}\right\}$ is of bounded geometry if and only if there exists $C \geq 1$ such that for all $i, j \in \mathbb{N}, i \neq j$

$$
\max \left\{\operatorname{diam}\left(\phi_{i}(X)\right), \operatorname{diam}\left(\phi_{j}(X)\right)\right\} \leq C \operatorname{dist}\left(\phi_{i}(X), \phi_{j}(X)\right) .
$$

The next theorem provides a necessary and sufficient condition for two systems of bounded geometry to be bi-Lipschitz equivalent.

Theorem 2.2. If both systems $\left\{f_{i}: X \rightarrow X: i \in \mathbb{N}\right\}$ and $\left\{g_{i}: Y \rightarrow Y: i \in \mathbb{N}\right\}$ are of bounded geometry, then the topological conjugacy $h: J_{f} \rightarrow J_{G}$ is biLipschitz continuous if and only if the following two conditions are satisfied.

(a) $Q^{-1} \leq \frac{\operatorname{diam}\left(f_{\omega}(X)\right)}{\operatorname{diam}\left(g_{\omega}(Y)\right)} \leq Q$ for some $Q \geq 1$ and all $\omega \in \mathbb{N}^{*}$. 
(b) $D^{-1} \leq \frac{\operatorname{dist}\left(g_{i}(Y), g_{j}(Y)\right)}{\operatorname{dist}\left(f_{i}(X), f_{j}(X)\right)} \leq D$ for some $D \geq 1$ and all $i, j \in \mathbb{N}, i \neq j$.

Proof. First notice that, due to the boundedness of geometry of $F$ and $G$, (a) and (b) remain true, with modified constants $Q$ and $D$ if necessary, if $X$ is replaced by $J_{F}$ and $Y$ is replaced by $J_{G}$ respectively. Suppose first that $x \in f_{i}\left(J_{F}\right)$ and $y \in f_{j}\left(J_{F}\right)$ with $i \neq j$. Then

$$
\begin{aligned}
|h(y)-h(x)| & \leq \operatorname{diam}\left(g_{i}\left(J_{G}\right)\right)+\operatorname{dist}\left(g_{i}\left(J_{G}\right), g_{j}\left(J_{G}\right)\right)+\operatorname{diam}\left(g_{j}\left(J_{G}\right)\right) \\
& \leq Q \operatorname{diam}\left(f_{i}\left(J_{F}\right)\right)+D \operatorname{dist}\left(f_{i}\left(J_{F}\right), f_{j}\left(J_{F}\right)\right)+Q \operatorname{diam}\left(f_{j}\left(J_{F}\right)\right) \\
& \leq 2 Q C \operatorname{dist}\left(f_{i}\left(J_{F}\right), f_{j}\left(J_{F}\right)\right)+D \operatorname{dist}\left(f_{i}\left(J_{F}\right), f_{j}\left(J_{F}\right)\right) \\
& \leq(2 Q C+D) \operatorname{dist}\left(f_{i}\left(J_{F}\right), f_{j}\left(J_{F}\right)\right) \\
& \leq(2 Q C+D)|y-x|
\end{aligned}
$$

Suppose in turn that $x \neq y$ both belong to the same element $f_{k}\left(J_{F}\right)$. Then there exist $\omega \in I^{*}(|\omega| \geq 1)$ and $i \neq j \in \mathbb{N}$ such that $x, y \in f_{\omega}\left(J_{F}\right), x \in$ $f_{\omega i}\left(J_{F}\right)$ and $y \in f_{\omega j}\left(J_{F}\right)$. From what has been proved so far we know that $\left|g_{\omega}^{-1}(h(y))-g_{\omega}^{-1}(h(x))\right| \leq(2 Q C+D)\left|f_{\omega}^{-1}(y)-f_{\omega}^{-1}(x)\right|$. Since $|y-x| \asymp$ ||$f_{\omega}^{\prime}||\left|f_{\omega}^{-1}(y)-f_{\omega}^{-1}(x)\right|$ and $|h(y)-h(x)| \asymp|| g_{\omega}^{\prime}||\left|g_{\omega}^{-1}(h(y))-g_{\omega}^{-1}(h(x))\right|$, we get

$$
|h(y)-h(x)| \leq \operatorname{const} \frac{\| g_{\omega}^{\prime} \mid}{\| f_{\omega}^{\prime} \mid}|y-x| \asymp|y-x|,
$$

where the comparability sign we have written due to (a) and equivalence of conditions (1) and (3) of Theorem 2.1. In the same way we show that $h^{-1}$ is Lipschitz continuous which completes the proof of the first part of our theorem.

So suppose now that $h$ is bi-Lipschitz continuous. We show that conditions (a) and (b) are satisfied. Indeed, to prove (a) suppose that $a$ and $b$ in $f_{\omega}\left(J_{F}\right)$ are taken so that $|h(a)-h(b)| \geq \frac{1}{2} \operatorname{diam}\left(g_{\omega}\left(J_{G}\right)\right)$. Then

$$
\operatorname{diam}\left(g_{\omega}\left(J_{G}\right)\right) \leq 2|h(a)-h(b)| \leq 2 L|a-b| \leq 2 L \operatorname{diam}\left(f_{\omega}\left(J_{F}\right)\right),
$$

where $L$ is a Lipschitz constant of $h$ and $h^{-1}$. In the same way it can be shown that $\operatorname{diam}\left(f_{\omega}\left(J_{F}\right)\right) \leq 2 L \operatorname{diam}\left(g_{\omega}\left(J_{G}\right)\right)$ which completes the proof of property (a). In order to prove the right-hand side of property (b) we proceed as follows. Fix $i, j \in \mathbb{N}, i \neq j$ and $a \neq b \in J_{F}$. Then

$$
\begin{aligned}
\operatorname{dist}\left(g_{i}(Y), g_{j}(Y)\right) & \leq \operatorname{dist}\left(g_{i}\left(J_{G}\right), g_{j}\left(J_{G}\right)\right) \leq\left|g_{i}(h(a))-g_{j}(h(b))\right| \\
& =\left|h\left(f_{i}(a)\right)-h\left(f_{j}(b)\right)\right| \leq L\left|f_{i}(a)-f_{j}(b)\right| \\
& \leq L\left(\operatorname{diam}\left(f_{i}(X)\right)+\operatorname{dist}\left(f_{i}(X), f_{j}(X)\right)+\operatorname{diam}\left(f_{j}(X)\right)\right) \\
& \leq L(2 C+1) \operatorname{dist}\left(f_{i}(X), f_{j}(X)\right),
\end{aligned}
$$


where the last inequality we wrote due to boundedness of geometry of the system $\left\{f_{i}: i \in \mathbb{N}\right\}$.

Remark 2.3. Notice that Theorem 2.1 and Theorem 2.2 remain true without assuming that the phase space $X$ is one-dimensional. We only need to know that the maps $f_{i}$ and $g_{i}$ are conformal and the assumption (2.7) of [MU] is satisfied.

Remark 2.4. Suppose now that the maps $i \mapsto \phi_{i}(X)$ are monotone; that is suppose that for all $i$ and $j, i<j$ implies $\phi_{i}(X)<\phi_{j}(X)$. We claim that, then the bounded geometry of the system is equivalent with the following weaker condition

$$
\max \left\{\operatorname { d i a m } \left(\phi_{i}(X), \operatorname{diam}\left(\phi_{i+1}(X)\right\} \leq C \operatorname{dist}\left(\phi_{i}(X), \phi_{i+1}(X)\right)\right.\right.
$$

Indeed, if $i<j$, then

$$
\begin{aligned}
\max \left\{\operatorname { d i a m } \left(\phi_{i}(X), \operatorname{diam}(\right.\right. & \left.\phi_{j}(X)\right\} \\
& \leq \max _{i \leq k \leq j-1}\left\{\max \left\{\operatorname{diam}\left(\phi_{k}(X)\right), \operatorname{diam}\left(\phi_{k+1}(X)\right)\right\}\right\} \\
& \leq \max _{i \leq k \leq j-1}\left\{C \operatorname{dist}\left(\phi_{k}(X), \phi_{k+1}(X)\right)\right\} \\
& \leq C \operatorname{dist}\left(\phi_{i}(X), \phi_{j}(X)\right),
\end{aligned}
$$

where writing the last inequality we used the monotonicity of the map $i \mapsto$ $\phi_{i}(X)$. The opposite implication is obvious.

Remark 2.5. If both maps $i \mapsto f_{i}(X)$ and $i \mapsto g_{i}(X)$ are monotone, then condition (b) of Theorem 2.2 can be replaced by the following.

(c) $C^{-1} \leq \frac{\operatorname{dist}\left(g_{k}(Y), g_{k+1}(Y)\right)}{\operatorname{dist}\left(f_{k}(X), f_{k+1}(X)\right)} \leq C$

for some constant $C \geq 1$ and all $k \in \mathbb{N}$. Indeed, assuming (c) this follows from 
the following computation.

$$
\begin{aligned}
\operatorname{dist}\left(g_{i}(Y), g_{j}(Y)\right)= & \sum_{k=i}^{j-1} \operatorname{dist}\left(g_{k}(Y), g_{k+1}(Y)\right)+\sum_{k=i+1}^{j-1} \operatorname{diam}\left(g_{k}(X)\right) \\
\leq & \sum_{k=i}^{j-1} C \operatorname{dist}\left(f_{k}(X), f_{k+1}(X)\right)+Q \sum_{k=i+1}^{j-1} \operatorname{diam}\left(f_{k}(X)\right) \\
\leq & \max \{C, Q\}\left(\sum_{k=i}^{j-1} \operatorname{dist}\left(f_{k}(X), f_{k+1}(X)\right)\right. \\
& \left.+\sum_{k=i+1}^{j-1} \operatorname{diam}\left(f_{k}(X)\right)\right) \\
= & \max \{C, Q\} \operatorname{dist}\left(f_{i}(X), f_{j}(X)\right)
\end{aligned}
$$

\section{Real-analytic Systems}

We call a 1-dimensional system $\Phi=\left\{\phi_{i}: X \rightarrow X, i \in \mathbb{N}\right\}$ real analytic if and only if there exists an open topological disk $D$ such that all the maps $\phi_{i}$ extend in a conformal (so 1-to-1) fashion to $D$ into $D$. Suppose that $\Phi$ is regular. Let $m$ be the conformal measure associated to the system $\Phi$ and let $\mu$ be the only probability invariant measure equivalent with $m$ (see [MU,Theorem 3.8], where this measure was denoted by $\left.\mu^{*}\right)$. We call the system $\Phi$ non-linear (comp. [S1]) if and only if at least one of the Jacobians $J_{\phi_{i}}=\frac{d \mu \circ \phi_{i}}{d \mu}$ is not constant. We shall prove the following theorem which is stronger than both Theorem 2.1 and Theorem 2.2.

Theorem 3.1. If both systems $\left\{f_{i}: X \rightarrow X: i \in \mathbb{N}\right\}$ and $\left\{g_{i}: Y \rightarrow Y: i \in \mathbb{N}\right\}$ are real-analytic, regular, and non-linear, then the following conditions are equivalent.

(a) The conjugacy between the systems $\left\{f_{i}: X \rightarrow X: i \in \mathbb{N}\right\}$ and $\left\{g_{i}: Y \rightarrow\right.$ $Y: i \in \mathbb{N}\}$ extends in a real-analytic fashion to the convex hull of $J_{F}$.

(b) The conjugacy between the systems $\left\{f_{i}: X \rightarrow X: i \in \mathbb{N}\right\}$ and $\left\{g_{i}: Y \rightarrow\right.$ $Y: i \in \mathbb{N}\}$ is bi-Lipschitz continuous.

(c) $\left|g_{\omega}^{\prime}\left(y_{\omega}\right)\right|=\left|f_{\omega}^{\prime}\left(x_{\omega}\right)\right|$ for all $\omega \in \mathbb{N}^{*}$, where $x_{\omega}$ and $y_{\omega}$ are the only fixed points of $f_{\omega}: X \rightarrow X$ and $g_{\omega}: Y \rightarrow Y$ respectively. 
(d) $\exists S \geq 1 \forall \omega \in \mathbb{N}^{*} S^{-1} \leq \frac{\operatorname{diam}\left(g_{\omega}(Y)\right)}{\operatorname{diam}\left(f_{\omega}(X)\right)} \leq S$.

(e) $\exists E \geq 1 \forall \omega \in \mathbb{N}^{*} E^{-1} \leq \frac{\left\|g_{\omega}^{\prime}\right\|}{\left\|f_{\omega}^{\prime}\right\|} \leq E$.

(f) $\operatorname{HD}\left(J_{G}\right)=\operatorname{HD}\left(J_{F}\right)$ and the measures $m_{G}$ and $m_{F} \circ h^{-1}$ are equivalent.

(g) The measures $m_{G}$ and $m_{F} \circ h^{-1}$ are equivalent.

Proof. The implication $(a) \Rightarrow(b)$ is obvious. That $(\mathrm{b}) \Rightarrow(\mathrm{c})$ results from the fact that (b) implies condition (1) of Theorem 2.1 which in view of this theorem is equivalent with condition (2) of Theorem 2.1 which finally is the same as condition (c) of Theorem 3.1. The implications (c) $\Rightarrow(\mathrm{d}) \Rightarrow(\mathrm{e}) \Rightarrow$ (f) have been proved in Theorem 2.1. The implication $(\mathrm{f}) \Rightarrow(\mathrm{g})$ is again obvious. We are left to prove that $(\mathrm{g}) \Rightarrow(\mathrm{a})$. As the first step we shall show that if a regular system $\left\{\phi_{i}: i \in \mathbb{N}\right\}$ is real-analytic, then the Jacobians $J_{\phi_{\omega}}$ of all the maps $\phi_{\omega}$, $\omega \in \mathbb{N}^{*}$ with respect to the invariant measure $\mu$ are also real analytic. Since $\frac{d\left(m \circ \phi_{\omega}\right)}{d m}=\left|\phi_{\omega}^{\prime}\right|^{h}$ and since $\phi_{\omega}^{\prime}\left\lceil_{X}\right.$ is a real-valued, either positive or negative, real-analytic function, the function $\left|\phi_{\omega}^{\prime}\right|^{h}$ is also real-analytic. Consequently, to check that

$$
\frac{d \mu \circ \phi_{\omega}}{d \mu}=\frac{d \mu \circ \phi_{\omega}}{d m \circ \phi_{\omega}} \cdot \frac{d m \circ \phi_{\omega}}{d m} \cdot \frac{d m}{d \mu}=\frac{d \mu}{d m} \circ \phi_{\omega} \cdot \frac{d m \circ \phi_{\omega}}{d m} \cdot \frac{d m}{d \mu}
$$

is real-analytic it suffices to check that $\frac{d \mu}{d m}$ is real-analytic. Let $D \subset \overline{\mathbb{C}}$ be the open topological disk claimed in the definition of real analytic systems. Since for each $\omega \in \mathbb{N}^{*},\left|\phi_{\omega}^{\prime} \|_{X}=_{-}^{+} \phi_{\omega}^{\prime}\right|_{X}$, all the derivatives extend (complex) analytically to the corresponding maps $\nu(\omega) \phi_{\omega}^{\prime}$, where $\nu(\omega) \in\{1,-1\}$. Given $n \geq 1$ consider the series of (complex) analytic functions $\mathcal{L}^{n}(1)=\sum_{|\omega|=n}\left(\nu(\omega) \phi_{\omega}^{\prime}\right)^{h}$, where $\left(\nu(\omega) \phi_{\omega}^{\prime}\right)^{h}$ are well-defined since $D$ is simply connected. Fix $x_{0} \in X$. By the Koebe Distortion Theorem and (3.3) of [MU] for all $n \geq 1$ and all $x \in D$ we can write

$$
\begin{aligned}
\left|\sum_{|\omega|=n}\left(\nu(\omega) \phi_{\omega}^{\prime}(x)\right)^{h}\right| & \leq \sum_{|\omega|=n}\left|\phi_{\omega}^{\prime}(x)\right|^{h} \leq K^{h} \sum_{|\omega|=n}\left|\phi_{\omega}^{\prime}\left(x_{0}\right)\right|^{h} \\
& =K^{h} \mathcal{L}^{n}(1)\left(x_{0}\right) \leq K^{2 h} .
\end{aligned}
$$

Hence, the maps $\mathcal{L}^{n}(1): D \rightarrow \mathbb{C}$ form a normal family in the sense of Montel. Since for $m$-a.e. point $x, \mathcal{L}^{n}(1)(x)$ converges to $\rho(x)=\frac{d \mu}{d m}(x)$, we conclude more, that $\left.\mathcal{L}^{n}(1)\right|_{D}$ converges to an analytic extension of $\rho$ on $D$. We will 
keep the same notation $\rho$ for this extension. So, we have proved that all the Jacobians $J_{\phi_{\omega}}=\frac{d \mu \circ \phi_{\omega}}{d \mu}$ are real-analytic, and in fact, extend analytically onto $D$. Now suppose that condition (g) of Theorem 3.1 is satisfied. Then $\mu_{F}=\mu_{G} \circ h$ meaning that $J_{h}=\frac{d \mu_{G} \circ h}{d \mu_{F}}=1$. Since $h \circ f_{\omega}=g_{\omega} \circ h$, the chain rule implies that $J_{h} \circ f_{\omega} \cdot J_{f_{\omega}}=J_{g_{\omega}} \circ h \cdot J_{h}$ and consequently $J_{f_{\omega}}=J_{g_{\omega}} \circ h$. Let now $g_{i}$ be a contraction produced by non-linearity. Notice, then that $J_{g_{i}}$ has only finitely many extremal points, since otherwise the equation $J_{g_{i}}^{\prime}=0$ would have an accumulation point in $Y$ which in turn would imply that $J_{g_{i}}$ would be constant on $Y$, contrary to the choice of $g_{i}$. Hence $J_{g_{i}}^{-1} \circ J_{f_{i}}$ is well-defined and 1-to-1 on an open set $V \subset X$, and $h=J_{g_{i}}^{-1} \circ J_{f_{i}}$ on $V \cap J_{F}$. Consider now $\omega \in \mathbb{N}^{*}$ such that $f_{\omega}(X) \subset V$. Denote by $C_{F}$ the convex hull of $J_{F}$. Then the map $g_{\omega}^{-1} \circ\left(J_{g_{i}}^{-1} \circ J_{f_{i}}\right) \circ f_{\omega}: C_{F} \rightarrow Y$ is well-defined, extends $h$, and is real-analytic.

\section{Scaling Functions}

From now on we assume that all our systems satisfy condition (a) of Lemma 2.2 of [MU]. This condition reads as follows.

There are two constants $L \geq 1$ and $\alpha>0$ such that

$$
|| \phi_{i}^{\prime}(y)|-| \phi_{i}^{\prime}(x)|| \leq L||\left(\phi_{i}^{\prime}\right)^{-1} \|^{-1}|y-x|^{\alpha},
$$

for every $i \in I$ and every pair of points $x, y \in V$. As a byproduct of the demonstration that $(\mathrm{b}) \Rightarrow$ (c) (p. 112 of $[\mathrm{MU}]$ ) we have shown that for all $\omega \in \mathbb{N}^{*}$, say $\omega \in \mathbb{N}^{n}$, and all $x, y \in X$

$$
|\log | \phi_{\omega}^{\prime}(y)|-\log | \phi_{\omega}^{\prime}(x)|| \leq \sum_{j=1}^{n} \|\left(\phi_{\omega_{j}}^{\prime}\right)^{-1}|| \cdot\left|\phi_{\omega_{j}}^{\prime}\left(y_{n-j}\right)\right|-\left|\phi_{\omega_{j}}^{\prime}\left(x_{n-j}\right)\right|,
$$

where $z_{k}=\phi_{\omega_{n-k+1}} \circ \cdots \circ \phi_{\omega_{n}}(z)$. In view of (4.1) this estimate continues as follows.

$$
\begin{aligned}
|\log | \phi_{\omega}^{\prime}(y)|-\log | \phi_{\omega}^{\prime}(x) \| & \leq \sum_{j=1}^{n} L\left|y_{n-j}-x_{n-j}\right|^{\alpha} \\
& \leq \sum_{j=0}^{n-1} L s^{j \alpha}|y-x|^{\alpha} \\
& =\frac{L}{1-s^{\alpha}}|y-x|^{\alpha}
\end{aligned}
$$


or equivalently

$$
\exp \left(\frac{-L}{1-s^{\alpha}}|y-x|^{\alpha}\right) \leq \frac{\left|\phi_{\omega}^{\prime}(y)\right|}{\left|\phi_{\omega}^{\prime}(x)\right|} \leq \exp \left(\frac{L}{1-s^{\alpha}}|y-x|^{\alpha}\right) .
$$

Now, since for every $t \geq 0$ sufficiently small $\left|e^{t}-1\right| \leq 2 t$, we get

$$
\begin{aligned}
|| \phi_{\omega}^{\prime}(y)|-| \phi_{\omega}^{\prime}(x)|| & =\left|\frac{\left|\phi_{\omega}^{\prime}(y)\right|}{\left|\phi_{\omega}^{\prime}(x)\right|}-1\right| \phi_{\omega}^{\prime}(x) \mid \\
& \leq \frac{2 L}{1-s^{\alpha}}|y-x|^{\alpha}\left|\phi_{\omega}^{\prime}(x)\right| \leq \frac{2 L s^{|\omega|}}{1-s^{\alpha}}|y-x|^{\alpha}
\end{aligned}
$$

In order to define scaling functions we will need the following basic lemma.

Lemma 4.1. If $\left\{\phi_{n}: X \rightarrow X: n \geq 1\right\}$ is a one-dimensional conformal iterated function system satisfying condition (4.1), then for every closed subinterval $K$ of $X$ and every $\omega \in \mathbb{N}^{\infty}$

$$
\lim _{n \rightarrow \infty} \frac{\left|\phi_{\omega_{n} \omega_{n-1} \ldots \omega_{0}}(K)\right|}{\left|\phi_{\omega_{n} \omega_{n-1} \ldots \omega_{0}}(X)\right|}:=S(\omega, K)
$$

exists and the convergence is uniform with respect to $K, n$ and $\omega$.

Proof. We shall show that the above sequence satisfies an appropriate Cauchy condition. So, fix $k<n$. We then have

$$
\begin{aligned}
\frac{\frac{\left|\phi_{\omega_{n} \ldots \omega_{k} \ldots \omega_{0}}(K)\right|}{\left|\phi_{\omega_{n} \ldots \omega_{k} \ldots \omega_{0}}(X)\right|}}{\frac{\left|\phi_{\omega_{k} \ldots \omega_{0}(K)}\right|}{\left|\phi_{\omega_{k} \ldots \omega_{0}}(X)\right|}} & =\frac{\frac{\left|\phi_{\omega_{n} \ldots \omega_{k+1}}\left(\phi_{\omega_{k} \ldots \omega_{0}}(K)\right)\right|}{\left|\phi_{\omega_{k} \ldots \omega_{0}}(K)\right|}}{\frac{\left|\phi_{\omega_{n} \ldots \omega_{k+1}}\left(\phi_{\omega_{k} \ldots \omega_{0}}(X)\right)\right|}{\left|\phi_{\omega_{k} \ldots \omega_{0}}(X)\right|}} \\
& =\frac{\left|\phi_{\omega_{n} \ldots \omega_{k+1}}^{\prime}\left(x_{n}\right)\right|}{\left|\phi_{\omega_{n} \ldots \omega_{k+1}}^{\prime}\left(y_{n}\right)\right|}
\end{aligned}
$$

for some $x_{n} \in \phi_{\omega_{k} \ldots \omega_{0}}(K)$ and $y_{n} \in \phi_{\omega_{k} \ldots \omega_{0}}(X)$, where the last equality sign we wrote due to the Mean Value Theorem. Denote now $\left|\phi_{\omega_{j} \ldots \omega_{0}}(K)\right| /\left|\phi_{\omega_{j} \ldots \omega_{0}}(X)\right|$ by $a_{j}$. In view of (4.5) and (4.2) we get

$$
\left|\log a_{n}-\log a_{k}\right| \leq \frac{L}{1-s^{\alpha}}\left|x_{n}-y_{n}\right|^{\alpha} \leq \frac{L}{1-s^{\alpha}}\left|\phi_{\omega_{k} \ldots \omega_{0}}(X)\right|^{\alpha} \leq \frac{L}{1-s^{\alpha}} s^{k \alpha} .
$$

Thus the sequence $\left\{\log a_{n}\right\}_{n=1}^{\infty}$ is a Cauchy sequence, and consequently $\left\{a_{n}\right\}_{n=1}^{\infty}$ itself is also a Cauchy sequence.

Let $\widetilde{\mathbb{N}}^{\infty}$ denote the set of infinite sequences of the form $\ldots \omega_{n} \omega_{n-1} \ldots \omega_{1} \omega_{0}$ and let $\widetilde{\mathbb{N}}^{*}$ denote the set of all finite words of the form $\omega_{n} \omega_{n-1} \ldots \omega_{1} \omega_{0}$. 
Lemma 4.1 allows us to introduce the scaling function (comp. also [S2] and $[\mathrm{PT}])$. In this section we will explore this notion. The weaker scaling function $S^{w}$ is defined on the space $\widetilde{\mathbb{N}}^{\infty} \times \mathbb{N}$, takes values in $(0,1)$, and is given by the formula

$$
S^{w}\left(\left\{\omega_{n}\right\}_{n=0}^{\infty}, i\right)=\lim _{n \rightarrow \infty} \frac{\left|\phi_{\omega_{n} \omega_{n-1} \ldots \omega_{0}}\left(\phi_{i}(X)\right)\right|}{\left|\phi_{\omega_{n} \omega_{n-1} \ldots \omega_{0}}(X)\right|},
$$

where the limit exists due to Lemma 4.1.

The stronger scaling function $S^{s}$ is defined similarly but on the larger space $\widetilde{\mathbb{N}} \times(\mathbb{N} \cup \mathcal{C})$, where $\mathcal{C}$ denotes the set of all connected components of $X \backslash \bigcup_{i=1}^{\infty} \phi_{i}(X)$. Frequently, given $\omega \in \mathbb{N}^{*}$ we will consider the function $S^{s}(\omega)$ : $(\mathbb{N} \cup \mathcal{C}) \rightarrow(0,1)$ given by the formula $S^{s}(\omega)(Z)=S^{s}(\omega, Z)$, and similarly we define the function $S^{w}(\omega)$. The following theorem is an immediate consequence of Lemma 4.1

Theorem 4.2. Both scaling functions $S^{w}: \widetilde{\mathbb{N}}^{\infty} \times \mathbb{N}$ and $S^{s}: \widetilde{\mathbb{N}} \times(\mathbb{N} \cup \mathcal{C})$ are continuous.

We now pass to consider two systems $F=\left\{f_{i}: i \in \mathbb{N}\right\}$ and $G=\left\{g_{i}: i \in\right.$ $\mathbb{N}$ \}. Our last theorem reads as follows.

Theorem 4.3. If the topological conjugacy $h: J_{F} \rightarrow J_{G}$ extends in a diffeomorphic fashion onto $X$, then $J_{F}$ and $J_{G}$ have the same strong scaling functions. If conversely, two topologically conjugate 1-dimensional i.f.s. $F$ and $G$ of bounded geometry have the same weak scaling functions and condition (b) of Theorem 2.2 is satisfied, then the topological conjugacy is bi-Lipschitz continuous.

Proof. Let us first prove the first part of this theorem. Indeed, let us keep the same notation $h$ for its diffeomorphic extension to $X$ and let $D$ be an arbitrary closed subinterval of $X$. For $\omega \in \widetilde{\mathbb{N}}^{\infty}$ we can write

$$
\frac{S(\omega, D)}{S(\omega, h(D))}=\lim _{n \rightarrow \infty} \frac{\frac{\left|f_{\omega_{n} \ldots \omega_{0}}(D)\right|}{\left|f_{\omega_{n} \ldots \omega_{0}}(X)\right|}}{\frac{\left|g_{\omega_{n} \ldots \omega_{0}}(h(D))\right|}{\left|g_{\omega_{n} \ldots \omega_{0}}(Y)\right|}}=\lim _{n \rightarrow \infty} \frac{\frac{\left|f_{\omega_{n} \ldots \omega_{0}}(D)\right|}{\left|g_{\omega_{n} \ldots \omega_{0}}(h(D))\right|}}{\frac{\left|f_{\omega_{n} \ldots \omega_{0}}(X)\right|}{\left|g_{\omega_{n} \ldots \omega_{0}}(Y)\right|}} .
$$

Now, by the Mean Value Theorem there exist $a_{n}$ and $b_{n}$ respectively in $f_{\omega_{n} \ldots \omega_{0}}(D)$ and in $f_{\omega_{n} \ldots \omega_{0}}(X)$ such that

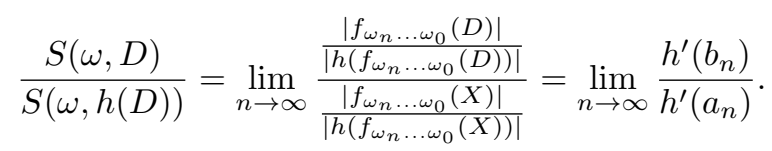


Since $h^{\prime}$ is uniformly continuous with no zeros and since $\left|b_{n}-a_{n}\right| \rightarrow 0$, the last limit is equal to 1 which finishes the proof of the first part of our theorem.

In order to show the second part of this theorem it suffices to show that condition (a) of Theorem 2.2 is satisfied. So, let $\tau=\tau_{0} \ldots \tau_{q-1}$ be an arbitrary word. Our aim is to show that $\left|\left(g_{\tau}\right)^{\prime}\left(h\left(x_{\tau}\right)\right)\right|=\left|\left(f_{\tau}\right)^{\prime}\left(x_{\tau}\right)\right|$, where $x_{\tau}$ is the only fixed point of the map $f_{\tau}: X \rightarrow X$. First notice that for every $n$

$\frac{\left|g_{\tau^{n+1} \tau_{0}}(Y)\right|}{\left|g_{\tau^{n} \tau_{0}}(Y)\right|}=\frac{\left|g_{\tau^{n+1}} \tau_{0}(Y)\right|}{\left|g_{\tau^{n+1}}(Y)\right|} \cdot \frac{\left|g_{\tau^{n+1}}(Y)\right|}{\left|g_{\tau^{n} \tau_{0} \ldots \tau_{q-2}}(Y)\right|} \cdot \frac{\left|g_{\tau^{n} \tau_{0} \ldots \tau_{q-2}}(Y)\right|}{\left|g_{\tau^{n} \tau_{0} \ldots \tau_{q-3}}(Y)\right|} \ldots . . \frac{\left|g_{\tau^{n} \tau_{0} \tau_{1}}(Y)\right|}{\left|g_{\tau^{n} \tau_{0}}(Y)\right|}$.

Hence

$$
\begin{aligned}
& \lim _{n \rightarrow \infty} \frac{\left|g_{\tau^{n+1} \tau_{0}}(Y)\right|}{\left|g_{\tau^{n} \tau_{0}}(Y)\right|} \\
& =S_{\tau^{\infty}}^{w}\left(\tau_{0}\right) S_{\tau^{\infty} \tau_{0} \ldots \tau_{q-2}}^{w}\left(\tau_{q-1}\right) S_{\tau^{\infty} \tau_{0} \ldots \tau_{q-3}}^{w}\left(\tau_{q-2}\right) \ldots S_{\tau^{\infty} \tau_{0}}^{w}\left(\tau_{1}\right)
\end{aligned}
$$

and similarly

$$
\begin{aligned}
& \lim _{n \rightarrow \infty} \frac{\left|f_{\tau^{n+1} \tau_{0}}(X)\right|}{\left|f_{\tau^{n} \tau_{0}}(X)\right|} \\
& =S_{\tau^{\infty}}^{w}\left(\tau_{0}\right) S_{\tau^{\infty} \tau_{0} \ldots \tau_{q-2}}^{w}\left(\tau_{q-1}\right) S_{\tau^{\infty} \tau_{0} \ldots \tau_{q-3}}^{w}\left(\tau_{q-2}\right) \ldots S_{\tau^{\infty} \tau_{0}}^{w}\left(\tau_{1}\right) .
\end{aligned}
$$

Since $g_{\tau^{n+1} \tau_{0}}(Y)=g_{\tau}\left(g_{\tau^{n} \tau_{0}}(Y)\right)$ and since $f_{\tau^{n+1} \tau_{0}}(X)=f_{\tau}\left(f_{\tau^{n} \tau_{0}}(X)\right)$, it follows from the Mean Value theorem that there exists $x_{n} \in f_{\tau^{n} \tau_{0}}(X)$ and $y_{n} \in g_{\tau^{n} \tau_{0}}(Y)$ such that $\left|g_{\tau^{n+1} \tau_{0}}(Y)\right|=\left|g_{\tau}^{\prime}\left(y_{n}\right)\right| \cdot\left|g_{\tau^{n} \tau_{0}}(Y)\right|$ and $\left|f_{\tau^{n+1} \tau_{0}}(X)\right|=$ $\left|f_{\tau}^{\prime}\left(y_{n}\right)\right| \cdot\left|f_{\tau^{n} \tau_{0}}(X)\right|$. Thus in view of our assumptions and (4.6) and (4.7) we get

$$
\lim _{n \rightarrow \infty} \frac{\left|g_{\tau}^{\prime}\left(y_{n}\right)\right|}{\left|f_{\tau}^{\prime}\left(x_{n}\right)\right|}=\lim _{n \rightarrow \infty} \frac{\frac{\left|g_{\tau^{n+1} \tau_{0}}(Y)\right|}{\left|g_{\tau^{n} \tau_{0}}(Y)\right|}}{\frac{\left|f_{\tau^{n}+1} \tau_{0}(X)\right|}{\mid f_{\tau} \tau_{0}}(X) \mid}=1 .
$$

Now, a straightforward observation shows that $y_{n} \rightarrow y_{\tau}$ and $x_{n} \rightarrow x_{\tau}$, where $y_{\tau}$ and $x_{\tau}$ are fixed points of $g_{\tau}$ and $f_{\tau}$ respectively. Hence $\left|g_{\tau}^{\prime}\left(y_{\tau}\right)\right|=\left|f_{\tau}^{\prime}\left(x_{\tau}\right)\right|$ and equivalence of this condition with condition (1) of Theorem 2.1 finishes the proof.

\section{References}

[MU] R. D. Mauldin, M. Urbański, Dimensions and measures in infinite iterated function systems, Proc. London Math. Soc. (3) 73 (1996), 105-154. 
[PT] F. Przytycki, F. Tangerman, Cantor sets in the line: Scaling functions of the shift map, Preprint 1992.

[S1] D. Sullivan, Quasiconformal homeomorphisms in dynamics, topology, and geometry, Proc. International Congress of Mathematicians, A.M.S. (1986), 1216-1228.

[S2] D. Sullivan, Differentiable structures on Fractal-like sets, Determined by Intrinsic Scaling functions on Dual Cantor sets, The Mathematical Heritage of Herman Weyl, A.S. Proc. Symp. Pure Math. 48 (1988). 